\title{
Animal models for hepatocellular carcinoma arising from alcoholic and metabolic liver diseases
}

\author{
Ken Liu' ${ }^{1,2,3}$, Jinbiao Chen ${ }^{2,3}$, Geoffrey W. McCaughan ${ }^{1,2,3}$ \\ ${ }^{1}$ A.W. Morrow Gastroenterology and Liver Centre, Royal Prince Alfred Hospital, Sydney, NSW 2050, Australia. \\ ${ }^{2}$ Sydney Medical School, University of Sydney, Sydney, NSW 2050, Australia. \\ ${ }^{3}$ Liver Injury and Cancer Program, Centenary Institute, Sydney, NSW 2050, Australia.
}

Correspondence to: Dr. Geoffrey W. McCaughan, MBBS PhD FRACP FAHMS, Head of Liver Injury and Cancer Program, Centenary Research Institute, A.W. Morrow Professor of Medicine, Director A.W. Morrow Gastroenterology and Liver Centre, Director Australian Liver Transplant Unit, Royal Prince Alfred Hospital, University of Sydney, Sydney, NSW 2050, Australia. E-mail: g.mccaughan@centenary.usyd.edu.au

How to cite this article: Liu K, Chen J, McCaughan GW. Animal models for hepatocellular carcinoma arising from alcoholic and metabolic liver diseases. Hepatoma Res 2020;6:7. http://dx.doi.org/10.20517/2394-5079.2019.39

Received: 1 Dec 2019 First Decision: 27 Jan 2020 Revised: 9 Feb 2020 Accepted: 17 Feb 2020 Published: 28 Feb 2020

Science Editor: Darrell Crawford Copy Editor: Jing-Wen Zhang Production Editor: Jing Yu

\begin{abstract}
Hepatocellular carcinoma (HCC) is a major and increasing cause of clinical and economic burden worldwide. Now that there are effective therapies to control or eradicate viral aetiologies, the landscape of HCC is changing with alcoholic and metabolic liver diseases becoming major catalysts. The pathogenesis of HCC is complex and incompletely understood, hampering improvements in therapy. Animal models are essential tools for advancing study on the cellular and molecular processes in HCC and for screening potential novel therapies. Many models of hepatocarcinogenesis have been established using various methods including genetic engineering, chemotoxic agents and dietary manipulation to direct implantation of tumour cells. However, none of these can accurately replicate all features found in human diseases. In this review, we provide an overview of different mouse models of HCC with a particular focus on cancer arising from alcoholic liver disease, non-alcoholic fatty liver disease and hereditary haemochromatosis. We also highlight their strengths and limitations and provide perspectives for future study.
\end{abstract}

Keywords: Hepatocellular carcinoma, animal models, mouse models, non-alcoholic fatty liver disease, alcohol, haemochromatosis 


\section{INTRODUCTION}

Hepatocellular carcinoma (HCC) is the most common type of primary liver cancer and ranks as the fifth most common incident cancer and the fourth most common cause of cancer-related death worldwide. Major causes for HCC include chronic liver disease such as infection with hepatitis B virus (HBV) or hepatitis C virus (HCV), alcoholic liver disease (ALD) and non-alcoholic fatty liver disease (NAFLD) ${ }^{[1]}$. Over $80 \%$ of the world's HCCs are found in less developed countries due to the influence of chronic HBV infection; however, the incidence and mortality are largely decreasing in these regions due to immunisation and antiviral therapy ${ }^{[2]}$. Instead, the burden of HCC is increasing in Western or developed countries due to the rise of NAFLD-associated HCC ${ }^{[3,4]}$. Indeed, NAFLD has either already become or is on the verge of becoming the leading cause of HCC in most Western countries ${ }^{[5-8]}$. Alarmingly, even in non-Western countries where viral hepatitis-related HCC predominates, the proportion of patients with HCC due to NAFLD is increasing at an exponential rate ${ }^{[9,10]}$. Moreover, with no effective pharmacologic agents to date, the burden of NAFLD is expected to rise further in the future.

The epidemiology of HCC in the context of ALD is poorly captured with heterogeneous geographic distribution ${ }^{[11]}$. However, current data show alcohol accounts for $21 \%$ of HCC cases globally, making it the third leading cause (behind HBV and HCV) and the leading cause in many regions ${ }^{[12]}$. The age-specific incidence rates for ALD-related HCC are also increasing.

Alongside NAFLD, hereditary haemochromatosis $(\mathrm{HH})$ is another metabolic liver disease impacted by HCC which deserves special mention. HCC accounts for up to $28 \%-45 \%$ of deaths in HH patients and the relative risk of HCC development in those with cirrhosis is greater than $200{ }^{[13]}$. HCC has also been described in $\mathrm{HH}$ patients without cirrhosis. Furthermore, iron has been implicated as a cofactor for HCC development in other liver diseases such as NAFLD ${ }^{[14]}$.

Therefore, with continuing improvements in global HBV vaccination coverage and effective therapies to control HBV and eradiate HCV, alcohol and metabolic liver diseases will take their place as the major contributors of hepatocarcinogenesis in the coming decades.

\section{WHY DO WE NEED ANIMAL MODELS?}

The biology of HCC is complex and incompletely understood with no single dominant molecular pathology. However, therapeutic approaches for primary intervention over the past ten years have resulted in numerous negative randomised controlled trials ${ }^{[15,16]}$. The current approved therapies for advanced disease prolong survival by only 2-3 months ${ }^{[17]}$. Thus, new targets for therapies are urgently needed.

Unlike other cancers, HCC can be diagnosed by imaging criteria alone and few patients $(<30 \%)$ are eligible for curative surgical resection or liver transplantation ${ }^{[18]}$. This has limited the availability of human HCC tissue samples for study. Indeed, the large number of human studies that have classified human HCC at the molecular level have almost exclusively used tissue from relatively early HCC obtained at hepatic resection or transplantation. Thus, animal models of more advanced HCC have proved to be crucial for investigating the genetic alterations, signalling pathways and microenvironment interactions involved in hepatocarcinogenesis. Importantly, they also allow for the evaluation of potential novel treatment paradigms and drugs in preclinical trials.

Although many animal models of HCC exist, this review focuses on mouse (Mus musculus) models, which are considered some of the best animal models for studying HCC owing to their compact size, short lifespan, breeding capacity and physiologic and genetic similarities to human biology ${ }^{[19]}$. After a brief overview of HCC mouse models, the review concentrates on mouse models for HCC arising from ALD, NAFLD and $\mathrm{HH}$. 


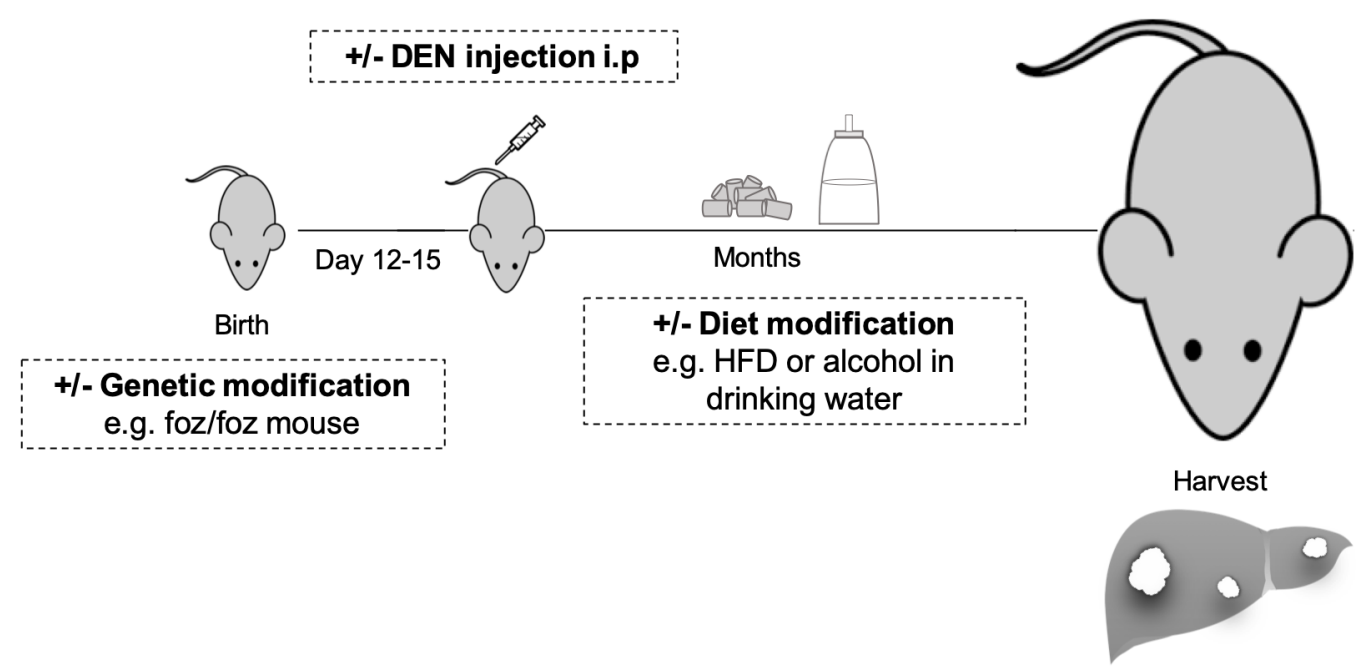

Figure 1. Examples of methodology used in mouse liver cancer models. DEN: di-ethyl-nitrosamine; HFD: high fat diet; i.p: intraperitoneal

\section{MOUSE MODELS FOR HCC (GENERAL APPROACHES)}

Hepatocarcinogenesis can be achieved through several different strategies either alone or in combination [Figure 1].

\section{Genetically engineered mouse models}

The most prevalent genetic mutations in human HCC are in the promoter region of TERT (60\%), TP53 (20\%-30\%), CTNNB1 (15\%-25\%), ARIDA1A (10\%-16\%) and AXIN1, while genes commonly mutated in other solid tumours such as EGFR, PIK3CA or KRAS are rarely mutated in HCC $(<5 \%)^{[20]}$. Various genetically engineered mouse (GEM) models have been created to reproduce these molecular features of human HCC. These models which result in activation of oncogenes or inactivation of tumour suppressor genes can be achieved via several different mechanisms including microinjection of recombinant DNA into the pronucleus of an embryo, lentiviral transduction in embryonic stem cells, homologous recombination in stem cells, conditional mutagenesis (e.g., Cre/loxP recombination system), knockdown using RNA interference and more recently genome editing with programmable endonucleases (e.g., CRISPR/Cas9 system). Liver-specific GEM models have also been created using the latter two techniques, for example with Albumin-Cre and hydrodynamic injection of plasmids, respectively ${ }^{[21]}$. Genetic modifications can also be used to produce mouse phenotypes that represent specific aetiologies of human metabolic liver diseases such as obese mice (e.g., ob/ob, db/db and foz/foz) to study NAFLD-related HCC or HFE knockout mice to study $\mathrm{HCC}$ in the setting of $\mathrm{HH}^{[22,23]}$.

However, the use of GEM models alone cannot recapitulate human disease. Firstly, there is no single dominant molecular pathology underlying all HCCs but rather several pathways involved ${ }^{[24]}$. Sequencing of cancer genomes had revealed that a typical cancer initiating cell accumulates at least 2-8 driver mutations $^{[25]}$. However, GEM models are generally limited to one specific driver mutation ${ }^{[21,26]}$, hence restricting models to study only specific genes or pathways in hepatocarcinogenesis. Secondly, GEM models typically lack chronic liver injury and fibrosis and HCCs develop in almost normal livers (with the notable exception of $M D R 2$ knockout mice). Despite this, these models have a role in providing evidence that powerful causation effects can be seen particularly following genetic ablation of key tumour suppressor genes or over amplification of oncogenic proteins. Examples of the former include liverspecific knockout of $p 53$ (AlfpCre $\operatorname{Trp}^{+} 3^{\Delta 2-10 / \Delta 2-10}$ mice), PTEN (AlbCrePten ${ }^{\text {flox/flox }}$ ) or both ${ }^{[27-29]}$. Conversely, overexpression of oncogenes such as $M Y C$ and $E_{2} F_{1}$ alone or synergistically in combination can also drive hepatocarcinogenesis ${ }^{[30,31]}$. Recently, Ruiz de Galarreta et al. ${ }^{[32]}$ were able to generate liver tumours with both MYC overexpression and TP53 depletion by hydrodynamic tail-vein injections of a transposon vector expressing MYC and a CRISPR/Cas9 vector expressing a single-guide RNA targeting Trp53 into C57BL/6 
mice. Another method is the use of stem cell transduction, which involves retroviral infection of hepatic progenitor cells isolated from foetal livers of mice to introduce oncogenes or target tumour suppressors into a healthy liver ${ }^{[21]}$. Manipulation of key genetic pathways in combination with liver injury models described below has now been advocated to achieve a more realistic representation of human HCC $^{[26]}$.

\section{Chemically- or diet-induced models}

Several chemotoxins can induce hepatocarcinogenesis by causing direct DNA damage (genotoxic) or promoting clonal expansion of preneoplastic cells (non-genotoxic). Di-ethyl-nitrosamine (DEN) is the most widely used genotoxic drug for chemically-induced HCC. Once bioactivated by cytochrome P450, DEN becomes an alkylating agent leading to the formation of mutagenic DNA adducts while also generating reactive oxygen species (ROS) which damage DNA, overall resulting in hepatocyte death. Similar to what occurs in humans, subsequent cycles of necrosis and regeneration in the mouse liver promote mutations, neoplastic transformation and eventual HCC development ${ }^{[33]}$. Indeed, DEN tumours have consistently exhibited high mutation rates ${ }^{[34]}$. DEN is most effective at inducing HCC when injected intraperitoneally into young male mice (less than two weeks old) when hepatocytes are still proliferating. Commonly used non-genotoxic carcinogens include carbon tetrachloride $\left(\mathrm{CCl}_{4}\right)$ and thioacetamide (TAA). These agents act as tumour promoters by damaging cellular structures, increasing the risk of genetic error and stimulating cell malignant transformation by affecting proliferation, differentiation and apoptosis processes ${ }^{[24]} \cdot \mathrm{CCl}_{4}$ is a potent hepatotoxin which causes centrilobular liver damage by the production of ROS and peroxidative degradation of phospholipids in plasma, lysosomal and mitochondrial membranes. Prolonged exposure (via oral, intraperitoneal or inhaled routes) leads to liver inflammation, fibrosis, cirrhosis and HCC development. TAA is another centrilobular hepatotoxin which can be administered via intraperitoneal injections or adding it to drinking water. It is bioactivated by mixed-function monooxygenases leading to its $S$-oxide and highly reactive $S, S$-dioxide, which modifies amine-lipids and proteins to initiate cellular necrosis ${ }^{[24]}$. The carcinogenic effects of all chemically-induced HCC models vary with age, mouse strain and sex. Ethanol feeding models are discussed in the ALD-associated HCC models section.

Diet-based models are most commonly used to study fatty liver diseases, particularly NAFLD and less so ALD. Mice are usually fed ad libitum with one of the following diets: high-fat diet (HFD), high-fat highcholesterol (HFHC), methionine and choline-deficient diet (MCD), choline-deficient high-fat diet (CDHFD), choline-deficient $L$-amino acid-defined (CDAA) diet or a Western diet (WD). Although these models can reliably produce steatosis, inflammation and even fibrosis, not many of them will result in HCC development after a prolonged period ${ }^{[22]}$. Furthermore, not all models reliably reproduce the accompanying metabolic features of the disease such as obesity and insulin resistance ${ }^{[21]}$. For example, a major drawback of the MCD model is that mice exhibit the opposite of the human metabolic syndrome with weight loss, no insulin resistance and low serum glucose, triglyceride and cholesterol. The specifics of these diet-based models and their combinations are further discussed in the NAFLD-associated HCC models section.

\section{Implantation models}

Human or murine HCC cell lines can be injected into recipient mice to form orthotopic tumours (intrahepatic, intrasplenic or intraportal injection) in the liver or heterotopic tumours (subcutaneous injection) typically in the flank. The main advantages of implantation models are their quick time to develop visible tumours (weeks to months in spontaneous models) that are easy to measure (especially subcutaneous heterotopic tumours) and reproducible - making them popular models for drug screening. This is counterbalanced by disadvantages such as considerable differences between cell lines necessitating multiple cell lines to be tested, the lack of tumour-liver microenvironment interactions in heterotopic models and the need for surgical expertise for orthotopic models ${ }^{[21]}$. Implantation of human cells (xenograft models) requires immunocompromised mice to prevent rejection of these foreign cells while murine cells can be implanted into immunocompetent mice (syngeneic/allograft models). Mouse tumour cell lines harbour mutations that are neutral or not relevant in human cancer making xenograft models more 
genetically applicable to human disease ${ }^{[35]}$. Patient-derived xenograft (PDX) models in which cells from a specific patient with HCC are transplanted into immunocompromised mice have been established ${ }^{[36]}$. PDXs faithfully recapitulate histologic, genomic and biological characteristics of the primary tumour and have been shown to predict drug response in HCC patients. However, this model is limited by engraftment failure rates of up to $60 \%$, long time to engraftment (several months) and high cost, which make it unsuitable for large-scale drug screening ${ }^{[36,37]}$. Furthermore, the major drawback of xenograft models (PDX or otherwise) is the lack of a tumoural immune response, which has become increasingly important as we enter the era of immunotherapies for HCC. Attempts at overcoming this with double humanised mouse models which express human hepatocytes and haematopoietic stem cells (and hence human immune cells) are technically intensive, expensive and not yet widely adopted ${ }^{[21]}$. Finally, xenograft of human HCC models which develop metastases (more readily than GEM models) have been established, providing the opportunity to study late-stage disease ${ }^{[38]}$.

\section{Replicability in human disease}

Human HCCs are highly complex and heterogeneous and thus cannot be adequately represented by any single mouse model. For example, gene expression profiles of tumours from the commonly-used DEN model was previously shown to be most similar to a subgroup of human HCC with poorer survival ${ }^{[39,40]}$. Correspondingly, many poor prognostic markers in human HCC are also highly expressed in DEN-induced tumours, e.g., alpha-foetoprotein (AFP). However, the DEN model lacks other hallmarks of human HCC, particularly fibrosis in the surrounding microenvironment ${ }^{[41]}$. In a more recent integrative genomic analysis of four separate mouse models and 987 human HCC samples, DEN tumours were found to be histologically hard to classify and least similar to human disease while Stelic Animal Model (STAM) tumours (discussed further in the NAFLD-associated HCC models section) were most molecularly similar to human HCC, especially high-grade, proliferative tumours with poor prognosis ${ }^{[34]}$. The authors further argued that DEN models should be avoided since they are dominated by mutational mechanisms not seen in human HCC. In contrast to DEN-induced and STAM tumours, $M D R 2$ knockout tumours are most similar to human HCCs associated with better survival ${ }^{[42]}$. However, the MDR2 knockout model produces a phenotype resembling humans with primary sclerosing cholangitis or primary biliary cholangitis rather than chronic "hepatitis" diseases caused by alcohol excess and HBV or HCV infection ${ }^{[43]}$. Very recently, experimental hepatocyte-specific activation of $\beta$-catenin also resulted the development of a phenotype that resembled the low proliferative subclass of human $\mathrm{HCC}^{[32]}$. Interestingly these tumours also had few intratumoural immune cells and were resistant to immune checkpoint inhibitor therapy. Therefore, it is clear that different models (and their combinations) are required to simulate specific subgroups of human HCC. Furthermore, drugs with known anti-tumour activity against human HCC do not demonstrate activity in some animal models and vice versa. Indeed, the current Food and Drug Administration-approved drugs for treating advanced HCC such as sorafenib and anti-programmed cell death receptor 1 (PD1) antibodies were trialled based on success in other cancers (advanced renal cell carcinoma and melanoma, respectively) rather than positive results in HCC animal models per se.

\section{SPECIFIC MOUSE MODELS FOR ALCOHOLIC AND METABOLIC LIVER DISEASE- ASSOCIATED HCC}

\section{ALD-associated HCC models}

In general, mice and other species (except the golden hamster) dislike alcohol and avoid ingestion when it is offered ad libitum ${ }^{[21,44]}$. Therefore, ALD mouse models are established by one of three ways: (1) replacing the food and water source with a liquid diet in which $5 \%$ ethanol accounts for $36 \%$ of total calories (LieberDeCarli model); (2) binge feeding mice with ethanol via gavage in addition to chronic ingestion [National Institute of Alcohol Abuse and Alcoholism (NIAAA) model]; or (3) intragastric ethanol infusion via a surgically inserted infusion pump (Tsukamoto-French model) [Table 1]. 


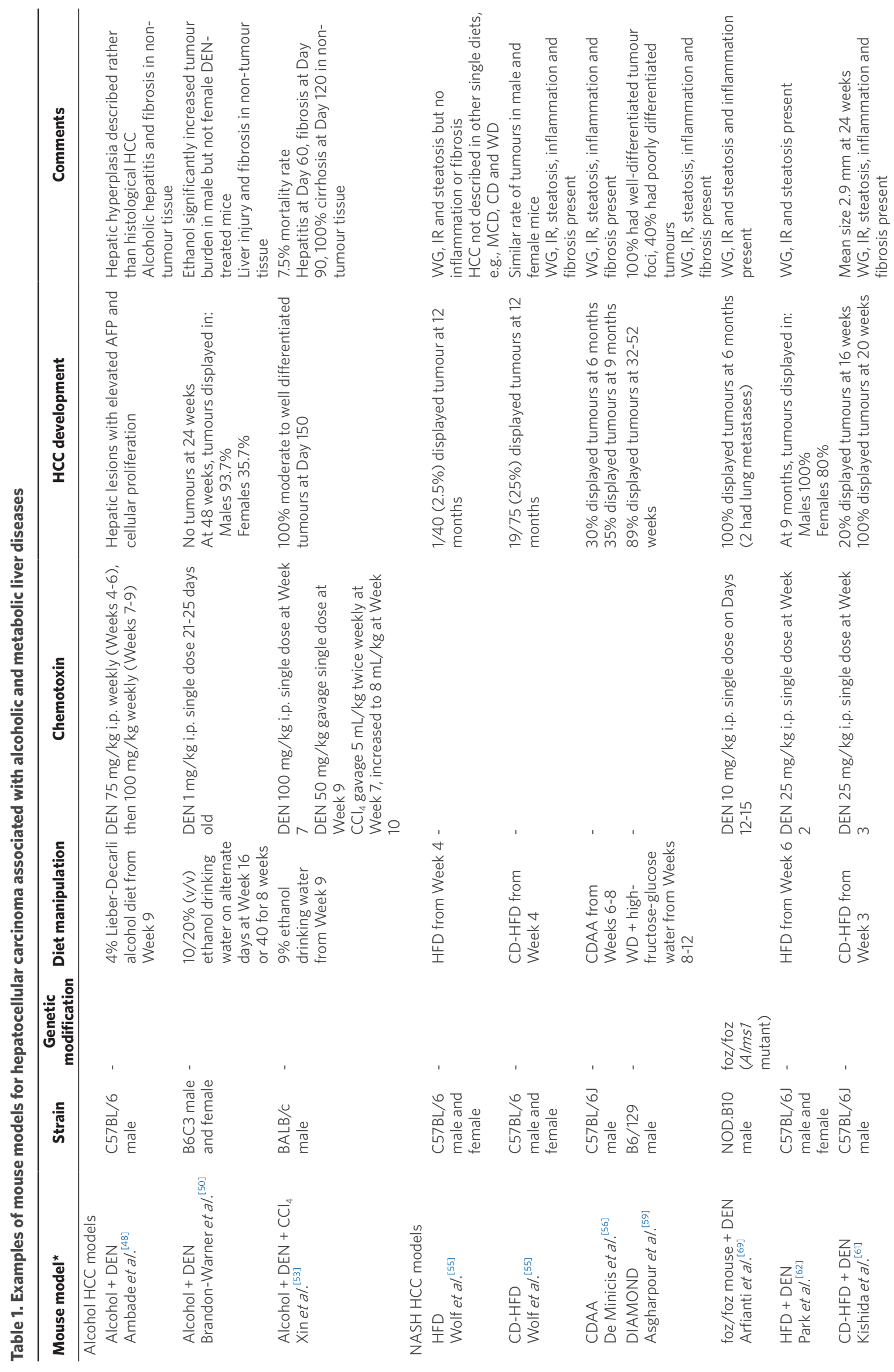




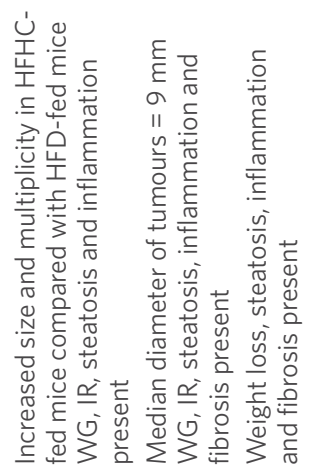

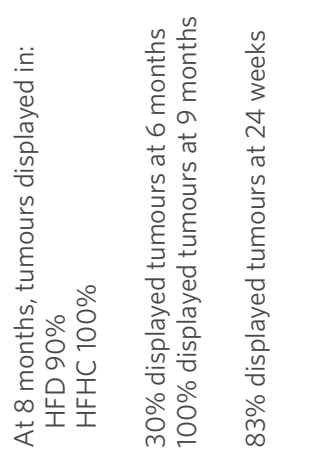
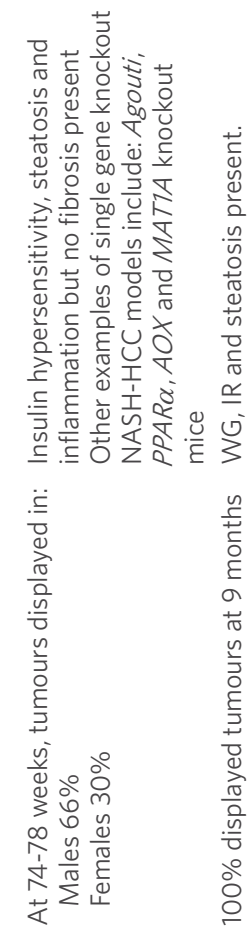
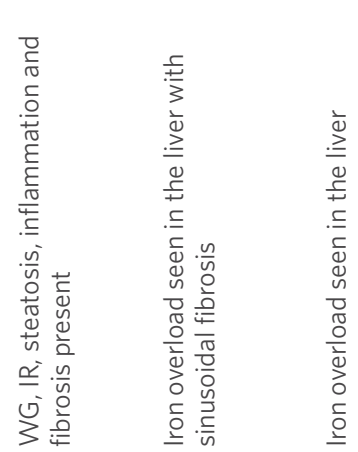

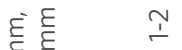

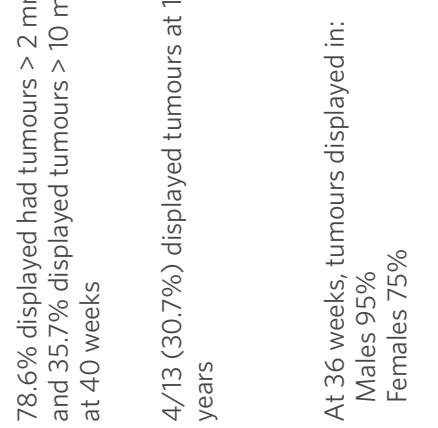

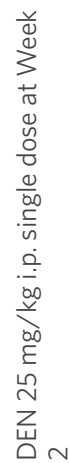

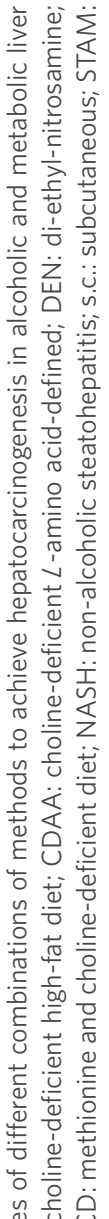

幽 든

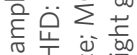

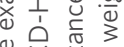

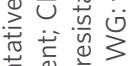

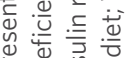

京

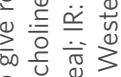

苦它总

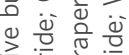

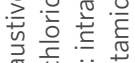

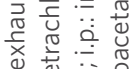

শ

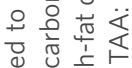

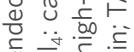

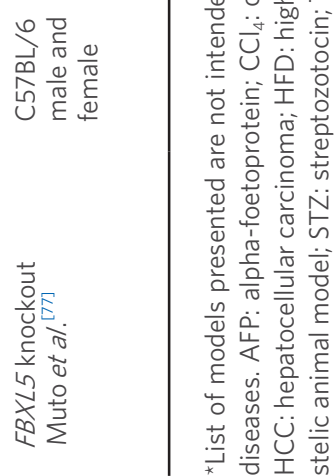


Aside from their natural aversion to alcohol, mice metabolise alcohol five times faster than humans ${ }^{[45]}$. As a result, the aforementioned ALD mouse models tend to exhibit less liver injury than seen in human disease $^{[21]}$. The Lieber-DeCarli model induces mild steatosis with little to no inflammation or fibrosis. The technically demanding Tsukamoto-French model produces severe steatosis but only mild inflammation and mild fibrosis. Although chronic or binge ethanol feeding regimens cause minor liver changes by themselves, their combination in the NIAAA model synergistically induces more severe steatosis and inflammation, with only mild chicken-wire fibrosis ${ }^{[46]}$.

Many have studied liver injury patterns of ALD mouse models; however, few have examined hepatocarcinogenesis specifically. As described above, the mild severity of liver inflammation and fibrosis induced by standalone mouse models of ALD means HCCs do not develop spontaneously. Therefore, a "second-hit" usually consisting of a chemical hepatotoxin is required for progression of ALD to cirrhosis and/or HCC. Indeed, of the hepatotoxins, DEN-induced C57BL/6 tumours have recently been matched to most resemble alcohol-induced HCC both morphologically and by comparative genomic hybridisation in a study comparing five different HCC models with human data ${ }^{[47]}$. Ambade et al. ${ }^{[48]}$ established a model of alcohol-driven HCC in adult C57BL/6 male mice. The four-week-old mice were administered six doses of DEN (or saline) intraperitoneally $(75 \mathrm{mg} / \mathrm{kg}$ weekly for three weeks and then $100 \mathrm{mg} / \mathrm{kg}$ weekly for three weeks) followed by the Lieber-DeCarli diet (or calorie-matched control diet) for seven weeks before sacrifice at 15 weeks. Compared to mice fed with a control diet, alcohol-fed mice had greater liver inflammation (raised alanine aminotransferase) and fibrosis. The alcohol-fed group also exhibited numerous liver nodules of hepatic hyperplasia associated with increased AFP expression and cellular proliferation, which the authors thought represented signs of early hepatocarcinogenesis. There were no hyperplastic nodules seen in the alcohol-fed saline-injected group or the control-fed DEN-injected group, thus confirming the need for a second stressor to initiate hepatocarcinogenesis. Early precancerous lesions were also described in another model using the combination of DEN and alcohol diet ${ }^{[4]}$. In this study, male C57BL/6 mice were injected intraperitoneally with DEN $(25 \mathrm{mg} / \mathrm{kg})$ at two weeks of age and then fed with the Lieber-DeCarli diet at eight weeks of age for 21 days. Over half of DEN-injected alcohol-fed mice developed precancerous basophilic foci compared to none in the DEN-injected control diet group. Interestingly, dietary luteolin (a flavonoid with anti-cancer properties) co-administration completely abrogated the development of precancerous lesions potentially by restoring sirtuin 1 activity and increasing downstream proliferator-activated receptor gamma coactivator 1 alpha protein expression. In a longer model, Brandon-Warner et al..$^{[50]}$ studied DEN-injected alcohol-fed B6C3 mice for 48 weeks and observed tumours in $94 \%$ and $36 \%$ of males and females, respectively. While chronic ethanol feeding exacerbated tumour formation in DEN-injected males, fewer and smaller tumours were observed in females exposed to ethanol compared to DEN-injected control-fed mice of respective sexes. Further analysis of liver mRNA revealed elevated SMAD3 in male compared to female mice in response to liver injury from DEN and alcohol, suggesting that increased TGF $\beta$-SMAD3 signalling may enhance HCC promotion. Indeed, gender disparity (males $>$ females) in liver cancer both in humans and in DEN-injected mice is well-recognised and may be related to sex differences in MyD88-dependent IL-6 production mediated by the protective effect of oestrogen ${ }^{[51]}$.

The combination of alcohol and $\mathrm{CCl}_{4}$ has also been experimented, although predominantly in rats. Weekly injections of $\mathrm{CCl}_{4}$ and alcohol administration through drinking water led to HCC after 104 weeks in mice ${ }^{[52]}$. The impact of chemical carcinogens on HCC formation appears to be additive. Recently, Xin et al. ${ }^{[53]}$ combined DEN (100 mg/kg intraperitoneal and $50 \mathrm{mg} / \mathrm{kg}$ gavage once each), $\mathrm{CCl}_{4}$ twice weekly and $9 \%$ alcohol as drinking water together in adult (seven-week-old) BALB/c mice. Multifocal HCC was noted only five months (150 days) after DEN injection. Tumours were moderate to highly differentiated and secreted AFP, resembling human HCC. Furthermore, there was no evidence of toxicity in this model as these mice survived until sacrifice. 


\section{NAFLD-associated HCC models}

Many models have been developed to represent NAFLD and non-alcoholic steatohepatitis (NASH), although, as aforementioned, not all of them exhibit features of metabolic syndrome. This is particularly important in NAFLD-related HCC since the presence of obesity and/or diabetes are themselves independent risk factors for the development of cancer $^{[54]}$.

Most dietary models of NAFLD (HFD, HFHC, MCD, WD and CD diet) rarely induce HCC development alone ${ }^{[22]}$. If spontaneous HCC does occur, it is time-consuming (e.g., 2.5\% for C57BL/6 mice fed HFD for 12 months $)^{[55]}$. Combination diets such as CD-HFD and CDAA have been shown to significantly increase rates of tumour formation, although overall rates are still low: 25\% after 12 months and 35\% after 9 months, respectively ${ }^{[55,56]}$. Indeed, these diets can recapitulate the key features of human NASH (including fibrosis) and metabolic syndrome more so than single diets. Susceptibility to tumour formation in dietary models also appears to be strain-dependent with DBA/2J $>\mathrm{C} 57 \mathrm{LBL} / 6>\mathrm{A} / \mathrm{J}^{[57,58]}$. Asgharpour et al.$^{[59]}$ generated an isogenic strain (B6/129) derived from a cross of two common mouse strains, C57BL/6J and 129S1/SvImJ, and fed them a high-fat-high-carbohydrate diet with high-fructose-glucose water - so-called DIAMOND mice. This promising model mimicked all the physiological, metabolic, histological and transcriptomic gene signature and clinical endpoints of human NASH including HCC in $89 \%$ at $32-52$ weeks. These tumours had gene signatures which strongly resembled the S1 and S2 human subclasses of HCC. Interestingly, neither C57BL/6J nor 129S1/SvImJ parent strain mice fed with the same diet developed HCC.

Combining dietary models with a hepatotoxin substantially hastens and increases HCC formation (i.e., up to $100 \%$ of male $\mathrm{C} 57 \mathrm{BL} / 6$ mice fed CDAA, HFD, CD-HFD or WD + intraperitoneal injections of DEN or $\mathrm{CCl}_{4}$ at 6-9 months) as well as tumour size ${ }^{[56,60-62]}$. The addition of cholesterol to a HFD (HFHC) in a DEN-induced model appears to further increase tumour burden ${ }^{[63]}$. In another model, Henderson et al ${ }^{[64]}$, treated male C57BL/6 mice with DEN (25 mg/kg once at 14 days old), TAA (300 mg/L in drinking water ad libitum from four weeks old) and HFD. These agents acted synergistically to develop HCCs in $83 \%$ of mice as early as 24 weeks of age, which was significantly more than control mice or those treated with DEN and TAA only. However, combining with hepatotoxins needs to be tempered by some limitations. For example, use of $\mathrm{CCl}_{4}$ can induce liver metabolism enzymes (which may impact the use of this model for drug discovery) and also mitigate metabolic processes involved in NASH, particularly susceptibility to diet-induced obesity and insulin resistance ${ }^{[65]}$. As mentioned above, the STAM mouse was recently shown to be the mouse model (out of four studied) that most closely resembles human HCC at a molecular level. Specifically, STAM tumours carried mutations of CTNNB1 at a rate comparable to human tumours, and (less frequently) mutations of TP53 - the most frequently altered genes in human $\mathrm{HCC}^{[34]}$. In contrast, CTNNB1 and TP53 were rarely mutated in DEN-induced tumours, which instead carried Hras, Braf and APC mutations rarely seen in human HCC. The STAM combination involves first treating neonatal C57BL/6 male mice with low-dose streptozotocin (STZ) at Day 2, which induces diabetes by causing death of pancreatic $\beta$ cells, resulting in lean mice with hypoinsulinaemia and hyperglycaemia, but no insulin resistance (the phenotype of type 1 diabetes) ${ }^{[66]}$. STZ is also a DNA alkylating agent (similar to DEN) with potential carcinogenic effects ${ }^{[6]]}$. When these mice are then fed with HFD, they develop weight gain, NASH by eight weeks, cirrhosis and HCC relatively quickly by $16-20$ weeks ${ }^{[66]}$. Takakura et al ${ }^{[6]}$ characterised STAM tumours at 20 weeks by clinical parameters used in human liver disease [i.e., Child-Turcotte-Pugh score and dynamic contrast-enhanced computed tomography (CT) measurements of HCCs]. Interestingly, the authors deduced that STAM mice had cirrhosis corresponding to Child-Turcotte-Pugh Class B (significant coagulopathy, occasional ascites, no encephalopathy and normal albumin and bilirubin) and tumours equivalent to Barcelona Clinic Liver Cancer Stage B (intermediate) or C (advanced) disease in humans. No HCCs develop when STZ is given alone, again pointing to the need for an additional stimulus. Female mice treated with the STAM regimen also fail to develop tumours, akin to the gender disparity seen in other models. 
Genetically obese mice with metabolic syndrome such as ob/ob (leptin deficient) db/db (leptin-receptor deficient), and foz/foz (mutated Alms1 gene) promote tumourigenesis in the presence of a secondary insult (e.g., DEN) but do not otherwise develop HCC spontaneously ${ }^{[62,67,69]}$. Furthermore, ob/ob and db/db mice fail to develop significant liver fibrosis or NASH histology without the addition of one of the dietary models above ${ }^{[21]}$. Park et al. ${ }^{[62]}$ utilised a dietary (HFD) and genetic (ob/ob) obesity model in combination with DEN to show that obesity (no matter how it was achieved) promoted the development of DEN-induced HCC in C57BL/6 mice by enhanced production of the pro-inflammatory cytokines IL- 6 and TNF. Many other genetic models have been developed to study NAFLD-associated HCC including PTEN knockout, $P P A R \alpha$ knockout, $A O X$ knockout, $K K-A y / a$ (agouti gene mutation) and MAT1A knockout mice. While they all reliably form HCC, they fail to recapitulate NASH itself (in $K K-A y / a$ mice) or its associated aspects such as obesity and metabolic syndrome (in PTEN, PPAR $\alpha, A O X$ and MAT1A knockout mice) ${ }^{[2,62,67]}$. As an example, PTEN knockout mice (which develop tumours between 40 and 78 weeks) are hyper-responsive to insulin instead of being insulin resistant. Unsurprisingly, gene expression signatures from PTEN knockout mice are markedly different from that of other NASH mouse models ${ }^{[28,70]}$. One promising genetic model of NASH-driven HCC is the MUP-uPA transgenic mouse combined with HFD ${ }^{[71]}$. MUP-uPA mice express high amounts of urokinase plasminogen activator in hepatocytes leading to hepatocyte-specific endoplasmic reticulum stress and liver damage. These mice exhibited weight gain, insulin resistance, classic signs of NASH (steatosis, inflammation, ballooning), fibrosis and, importantly, spontaneous HCC in $80 \%$ at 40 weeks via processes dependent on TNF produced by inflammatory liver macrophages ${ }^{[7]}$. As expected, HFD-fed wild type mice developed simple steatosis and no HCC over the same period. Furthermore, transcriptomic data from MUP-uPA mice and human NASH datasets showed signalling similarities, especially in the regulation of the immune system, innate immune response and the response to cytokine gene sets ${ }^{[67]}$. Recently, Shalapour et al. ${ }^{[72]}$ used both MUP-uPA and STAM mice fed with HFD to make a landmark discovery that hepatocarcinogenesis in NASH was facilitated by immunosuppressive liverresident Ig $\mathrm{A}^{+}$plasma cells, which directly inhibit anti-tumour cytotoxic CD8+ T lymphocyte activation.

Of the models mentioned above, it seems the MUP-uPA and DIAMOND mice (which require a combination of genetic modification and dietary manipulation) best replicate NASH-associated HCC. However, tumour formation in these models requires lengthy periods and there is considerable heterogeneity in their mutational landscapes which may limit utility and reliability in some settings, e.g., drug development studies ${ }^{[67]}$. Although STAM mice can develop tumours more quickly than these models (20 weeks vs. 40 weeks), they are physiologically less similar to human NASH (lacking insulin resistance).

\section{HH-associated HCC models}

Hepatocarcinogenesis arising from iron accumulation is thought to be secondary to oxidative DNA damage from ROS generated by free hepatic iron. This leads to a cycle of cell death, and compensatory proliferation, which favours the accumulation of mutations in hepatocytes and ultimately malignant transformation ${ }^{[13,73]}$. Recreating this in an animal model is difficult. The most common form of $\mathrm{HH}$ is caused by mutations in the HFE gene. Although HFE gene knockout produces the phenotype of $\mathrm{HH}$ in mice, spontaneous liver tumours do not develop ${ }^{[74]}$. In a dietary model where BALB/cJ male mice were fed ad libitum with chow supplemented with 3\% carbonyl-iron, hepatic iron concentrations at 12 months were 13-fold that of normal chow-fed controls ${ }^{[75]}$. No liver tumours developed; however, hepatocyte nuclei changes were observed (iron-containing ferritin inclusions, enlarged nucleus, increased mitotic index and abnormal mitotic figures), which may have represented preneoplastic changes. Rothenberg et al. ${ }^{[76]}$ created a model of $\mathrm{HH}$ by knocking out $\beta 2$-microglobulin (the chaperone protein for HFE) in C57BL/6 mice and reported that spontaneous HCCs developed in only a minority (31\%) of mice. Because tumour development was not predictable and time-consuming (taking up to two years), this model has not been widely used to study HH-related HCC. Recently, Muto et al. ${ }^{[7]}$ developed a novel model of HCC induced by iron overload by deleting the iron-sensing ubiquitin ligase FBXL5 specifically in hepatocytes and 
exposure to DEN. Alb-Cre/Fbxl $5^{\text {flox/flox }}$ mice were injected with DEN $(25 \mathrm{mg} / \mathrm{kg})$ intraperitoneally at Day 15 and tumours were significantly increased in number and size compared to DEN-injected control mice at 36 weeks in both males and females. The study demonstrated FBXL5 deficiency led to a sequence of events (iron overload, oxidative stress, liver damage and regenerative proliferation), which, with the addition of DEN, gave rise to liver tumours with high mutational load. Previously, hepatocyte-specific FBXL5 deletion without the addition of DEN was shown to cause liver inflammation but not tumours. The authors went on to analyse FBXL5 mRNA expression in five different human HCC cohorts and found that low FBXL5 expression level was indeed strongly associated with poorer prognosis in human HCC. Finally, the impact of iron on hepatocarcinogenesis has also been evaluated using a xenograft model. In this study, 3-4-weekold female BALB/c athymic mice (nu/nu) were injected subcutaneously with human HCC cell lines (HepзB or HepG2) and followed for 21 days ${ }^{[23]}$. The authors showed that TSC24 (a potent iron chelator) suppressed tumour growth in a dose-dependent manner by reducing available iron, and triggering cell-cycle arrest and apoptosis.

\section{THE ROLE OF THE GUT MICROBIOME}

Increasingly, the role of the gut microbiome has been implicated in alcoholic and metabolic liver diseases and HCC via the gut-liver axis, which refers to bidirectional communication between the gut (and its microbiome) and the liver ${ }^{[78]}$. In one direction, the liver secretes bile acids and antibodies into the intestine, which influences the gut microbiome composition. Reciprocally, the microbiome and its metabolites translocate the gut to reach the liver via the portal vein (the enterohepatic circulation) and regulate metabolic functions. This gut-liver axis exists in a homeostasis, which becomes disrupted in metabolic liver diseases.

Bacterial dysbiosis has been consistently demonstrated in the gut microbiomes of patients and mice with metabolic liver diseases and $\mathrm{HCC}^{[79]}$. Mouse model studies have already revealed several mechanisms by which the gut microbiome contributes to HCC development.

\section{Bacterial metabolism of compounds}

In a model of NASH-associated HCC, Yoshimoto et al. ${ }^{[80]}$ induced HCC by treatment with a chemical carcinogen [dimethylbenz (a)anthracene] and HFD. The authors found a strong increase in Gram-positive bacteria (particularly Clostridium spp.) as well as levels of deoxycholic acid (DCA), a secondary bile acid whose production relies on metabolism of primary bile acids by bacteria such as Clostridium. Significantly, DCA was shown to promote a senescence-associated secretory phenotype in hepatic stellate cells, which leads to hepatocarcinogenesis via activation of the TLR2 pathway ${ }^{[79,80]}$.

\section{Leaky gut}

Increased levels of lipopolysaccharide in the systemic circulation (due to increased intestinal permeability) and its interaction with TLR4 have been demonstrated to promote HCC formation in a CD-HFD-fed NASH model as well as a chemotoxin model with combination DEN and $\mathrm{CCl}_{4}^{[8,82]}$. This process can be abrogated by gut sterilisation with oral antibiotics, especially in late-stage disease.

\section{Immunosuppressive microenvironment}

The gut microbiome also modulates tumoural adaptive immune responses. The aforementioned study by Shalapour et al. ${ }^{[72]}$ showed that manipulating the gut microbiome in mice with NASH-driven HCC either by knocking out their polyimmunoglobulin receptor (which regulates IgA transport into the gut lumen and maintains microbial homeostasis) or giving them broad-spectrum antibiotics (which reduces gut bacterial load) promoted and inhibited HCC development, respectively. Both these interventions modulate liver and circulating IgA levels and hence anti-tumour cytotoxic T cell activation, as discussed above. 
The gut microbiome (and its associated HCC risk) can be transmissible between mice and, interestingly, this risk can also be transferred via the microbiome across generation to offspring of treated mothers ${ }^{[79,83]}$. This opens up another avenue to induce hepatocarcinogenesis alongside GEM, hepatotoxins and dietary manipulation in future models.

Thus, as we explore the new frontier of gut microbiome, animal models will be crucial for understanding causality, pathogenesis and testing of therapeutic options targeting the microbiome (e.g., antibiotics, probiotics, synthetic bile acids and faecal microbiota transplantation). Although mouse and human gut microbiome communities are dominated by the same set of bacterial phyla, they are on the whole distinct from one another ${ }^{[78]}$. Therefore, experimental findings from microbiome studies in mouse models need validation in human studies. The emerging use of a humanised gnotobiotic model (human donor stool transplanted into germ-free mice) may also improve the applicability of preclinical findings ${ }^{[84]}$.

\section{CURRENT CHALLENGES AND FUTURE DIRECTIONS}

Although, as described above, there are many different animal models for HCC related to alcoholic and metabolic liver diseases, a single model faithfully recapitulating all features of human disease is lacking and unlikely to exist. This is partly because human HCC is genetically heterogeneous, consisting of several subtypes that are clearly different in behaviour, prognosis and response to treatment themselves. Clearly, the identification of models that represent different human HCC subsets is required. Yan et al. ${ }^{[26]}$ argued for combining a chronic injury model (e.g., $\mathrm{NASH}, \mathrm{CCl}_{4}$ or $M D R 2$ knockout) with alterations in oncogenes or tumour suppressor genes found in human HCC which alone are not sufficient to cause hepatocarcinogenesis (e.g., weak activation of pathways by heterozygous deletion or targeting only a small percentage of hepatocytes) to achieve a more realistic representation of human HCC. The optimal combinations for each aetiology are yet to be determined and will be an area of further research. When achieved, this would not only help improve our understanding of the pathobiology of aetiology-specific HCC but also improve our preclinical testing of new targeted treatments as we work towards personalised medicine. Humanised mouse models may be a bridge for translating findings from mouse studies to humans and presents a promising future strategy. However, several major challenges need to be overcome not the least of which is the engraftment of a humanised immune system.

The amount of time required for tumourigenesis is another obstacle, as most models take more than nine months to produce macroscopic HCCs. Furthermore, time is also needed to establish steatosis, inflammation, fibrosis and cirrhosis ${ }^{[21]}$. While implantation HCC models are established within weeks, they are lacking these biologically important changes in the background liver. Indeed, human liver disease typically takes decades to progress to cirrhosis and HCC. For example, patients with NASH progress at a mean rate of only 0.09-0.14 fibrosis stages per year ${ }^{[54]}$. Thus, the models most representative of human HCC may require the most time which is suboptimal for studying response to therapy.

At present, almost all mouse studies assess tumour size and number at the one time point of sacrifice; however, in clinical practice, HCC is diagnosed and monitored regularly using imaging (CT, magnetic resonance imaging and ultrasound). Although these imaging modalities give reliable measurements that correlate with tumour size at sacrifice, they are currently time-consuming and labour intensive (requiring scanners, anaesthesia and injection of intravenous contrast agents) ${ }^{[21]}$. Since tumour development can be lengthy and their responses to treatment (especially new immunotherapies) are dynamic over time ${ }^{[85]}$, measurement of experimental tumours on imaging will likely play an increasingly important role in the future.

Recently, three-dimensional in vitro cell culture systems (organoids) using cells isolated from human biopsies have been developed to study HCC. These tumour organoids (tumouroids) have been shown to 
recapitulate the histological architecture, expression profile, genomic landscape and in vivo tumourigenesis of the parental tumour, even after long-term (>1 year) expansion in culture ${ }^{[37]}$. Furthermore, tumouroids could be established within 2-3 months after isolation. Therefore, tumouroids fulfil many of the criteria for a reliable cancer model which animal models could not and may represent a promising advancement for understanding tumour biology and drug efficacy testing in future studies of HCC. However, they currently lack the human immune and stromal microenvironment that is thought to be crucial in understanding tumour progression and response to treatment, particularly immune-based therapies.

\section{CONCLUSION}

Alcoholic and metabolic liver diseases will be major contributors to HCC burden in the future. Many aspects of human HCC development and progression remain unknown, negatively impacting therapeutic advancement. Animal models play a crucial role in improving our understanding of human HCC and developing novel therapeutic strategies. Currently, no animal model can faithfully replicate the complexity of the cancer and its background liver disease but mere aspects of it with varying degrees of technical demand. The careful combination of different animal models and use of novel technologies such as human organoids may help bridge this gap in the future. For the time being, the use of HCC mouse models needs to be tailored to specific experimental hypothesis or clinical testing.

\section{DECLARATIONS}

\section{Authors' contributions}

Acquisition, analysis and interpretation of data, drafting of the article, critical revision of the article: Liu K, Chen J, McCaughan GW

All authors have read and approved the final version.

\section{Availability of data and materials}

Not applicable.

\section{Financial support and sponsorship}

None.

\section{Conflicts of interest}

All authors declared that there are no conflicts of interest.

\section{Ethical approval and consent to participate}

Not applicable.

\section{Consent for publication}

Not applicable.

\section{Copyright}

(c) The Author(s) 2020.

\section{REFERENCES}

1. El-Serag HB. Hepatocellular carcinoma. N Engl J Med 2011;365:1118-27.

2. Wallace MC, Preen D, Jeffrey GP, Adams LA. The evolving epidemiology of hepatocellular carcinoma: a global perspective. Expert Rev Gastroenterol Hepatol 2015;9:765-79.

3. Wong MC, Jiang JY, Goggins WB, Liang M, Fang Y, et al. International incidence and mortality trends of liver cancer: a global profile. Sci Rep 2017;7:45846.

4. Bertuccio P, Turati F, Carioli G, Rodriguez T, La Vecchia C, et al. Global trends and predictions in hepatocellular carcinoma mortality. J 
Hepatol 2017;67:302-9.

5. Rinella M, Charlton M. The globalization of nonalcoholic fatty liver disease: Prevalence and impact on world health. Hepatology 2016;64:19-22.

6. Loomba R, Sanyal AJ. The global NAFLD epidemic. Nat Rev Gastroenterol Hepatol 2013;10:686-90.

7. Ertle J, Dechêne A, Sowa JP, Penndorf V, Herzer K, et al. Non-alcoholic fatty liver disease progresses to hepatocellular carcinoma in the absence of apparent cirrhosis. Int J Cancer 2011;128:2436-43.

8. Dyson J, Jaques B, Chattopadyhay D, Lochan R, Graham J, et al. Hepatocellular cancer: the impact of obesity, type 2 diabetes and a multidisciplinary team. J Hepatol 2014;60:110-7.

9. Cho EJ, Kwack MS, Jang ES, You SJ, Lee JH, et al. Relative etiological role of prior hepatitis B virus infection and nonalcoholic fatty liver disease in the development of non-B non-C hepatocellular carcinoma in a hepatitis B-endemic area. Digestion 2011;84:17-22.

10. Okanoue T, Umemura A, Yasui K, Itoh Y. Nonalcoholic fatty liver disease and nonalcoholic steatohepatitis in Japan. J Gastroenterol Hepatol 2011;26:153-62.

11. Ganne-Carrié N, Nahon P. Hepatocellular carcinoma in the setting of alcohol-related liver disease. J Hepatol 2019;70:284-93.

12. Global Burden of Disease Liver Cancer Collaboration. The burden of primary liver cancer and underlying etiologies from 1990 to 2015 at the global, regional, and national level results from the global burden of disease study 2015. JAMA Oncol 2017;3:1683-91.

13. Deugnier Y, Turlin B. Iron and hepatocellular carcinoma. J Gastroenterol Hepatol 2001;16:491-4.

14. Sorrentino P, D’Angelo S, Ferbo U, Micheli P, Bracigliano A, et al. Liver iron excess in patients with hepatocellular carcinoma developed on non-alcoholic steato-hepatitis. J Hepatol 2009;50:351-7.

15. Thillai K, Ross P, Sarker D. Molecularly targeted therapy for advanced hepatocellular carcinoma - a drug development crisis? World J Gastrointest Oncol 2016;8:173-85.

16. Nault JC. The end of almost 10 years of negative RCTs in advanced hepatocellular carcinoma. Lancet 2017;389:4-6.

17. Llovet JM, Ricci S, Mazzaferro V, Hilgard P, Gane E, et al. Sorafenib in advanced hepatocellular carcinoma. N Engl J Med 2008;359:378-90.

18. Forner A, Reig M, Bruix J. Hepatocellullar carcinoma. Lancet 2018;391:1301-14.

19. Newell P, Villanueva A, Friedman SL, Koike K, Llovet JM. Experimental models of hepatocellular carcinoma. J Hepatol 2008;48:858-79.

20. Bruix J, Han KH, Gores G, Llovet JM, Mazzaferro V. Liver cancer: approaching a personalized care. J Hepatol 2015;62:S144-56.

21. Brown ZJ, Heinrich B, Greten TF. Mouse models of hepatocellular carcinoma: an overview and highlights for immunotherapy research. Nat Rev Gastroenterol Hepatol 2018;15:536-54.

22. Lau JKC, Zhang X, Yu J. Animal models of non-alcoholic fatty liver disease: current perspectives and recent advances. J Pathol 2017;241:36-44

23. Ba Q, Hao M, Huang H, Hou J, Ge A, et al. Iron deprivation suppresses hepatocellular carcinoma growth in experimental studies. Clin Cancer Res 2011;17:7625-33.

24. Santos NP, Colaço AA, Oliveira PA. Animal models as a tool in hepatocellular carcinoma research: a review. Tumour Biol 2017;39:1-20.

25. Vogelstein B, Papadopoulos N, Velculescu VE, Zhou S, Diaz Jr LA, et al. Cancer genome landscapes. Science 2013;339:1546-58.

26. Yan J, Caviglia JM, Schwabe RF. Animal models of HCC - when injury meets mutation. J Hepatol 2018;68:193-4.

27. Liu Y, Qi X, Zeng Z, Wang L, Wang J, et al. CRISPR/Cas9-mediated p53 and Pten dual mutation accelerates hepatocarcinogenesis in adult hepatitis B virus transgenic mice. Sci Rep 2017;7:2796.

28. Horie Y, Suzuki A, Kataoka E, Sasaki T, Hamada K, et al. Hepatocyte-specific Pten deficiency results in steatohepatitis and hepatocellular carcinomas. J Clin Invest 2004;113:1774-83.

29. Katz SF, Lechel A, Obenauf AC, Begus-Nahrmann Y, Kraus JM, et al. Disruption of Trp53 in livers of mice induces formation of carcinomas with bilineal differentiation. Gastroenterology 2012;142:1229-39.e3.

30. Santoni-Rugiu E, Jensen MR, Thorgeirsson SS. Disruption of the $\mathrm{pRb} / \mathrm{E} 2 \mathrm{~F}$ pathway and inhibition of apoptosis are major oncogenic events in liver constitutively expressing c-myc and transforming growth factor alpha. Cancer Res 1998;58:123-34.

31. Kent LN, Bae S, Tsai SY, Tang X, Srivastava A, et al. Dosage-dependent copy number gains in E2f1 and E2f3 drive hepatocellular carcinoma. J Clin Invest 2017;127:830-42.

32. Ruiz de Galarreta M, Bresnahan E, Molina-Sánchez P, Lindblad KE, Maier B, et al. $\beta$-catenin activation promotes immune escape and resistance to anti-PD-1 therapy in hepatocellular carcinoma. Cancer Discov 2019;9:1124-41.

33. Bakiri L, Wagner EF. Mouse models for liver cancer. Mol Oncol 2013;7:206-23.

34. Dow M, Pyke RM, Tsui BY, Alexandrov LB, Nakagawa H, et al. Integrative genomic analysis of mouse and human hepatocellular carcinoma. Proc Natl Acad Sci U S A 2018;115:E9879-88.

35. Fidler IJ. Rationale and methods for the use of nude mice to study the biology and therapy of human cancer metastasis. Cancer Metastasis Rev 1986;5:29-49.

36. He S, Hu B, Li C, Lin P, Tang WG, et al. PDXliver: a database of liver cancer patient derived xenograft mouse models. BMC Cancer 2018;18:550.

37. Broutier L, Mastrogiovanni G, Verstegen MM, Francies HE, Gavarró LM, et al. Human primary liver cancer-derived organoid cultures for disease modeling and drug screening. Nat Med 2017;23:1424-35.

38. Sun FX, Tang ZY, Lui KD, Ye SL, Xue Q, et al. Establishment of a metastatic model of human hepatocellular carcinoma in nude mice via orthotopic implantation of histologically intact tissues. Int J Cancer 1996;66:239-43.

39. Calvisi DF, Ladu S, Gorden A, Farina M, Conner EA, et al. Ubiquitous activation of Ras and Jak/Stat pathways in human HCC. Gastroenterology 2006;130:1117-28.

40. Lee JS, Chu IS, Mikaelyan A, Calvisi DF, Heo J, et al. Application of comparative functional genomics to identify best-fit mouse models 
to study human cancer. Nat Genet 2004;36:1306-11.

41. Inokuchi S, Aoyama T, Miura K, Osterreicher CH, Kodama Y, et al. Disruption of TAK1 in hepatocytes causes hepatic injury, inflammation, fibrosis, and carcinogenesis. Proc Natl Acad Sci U S A 2010;107:844-9.

42. Katzenellenbogen M, Mizrahi L, Pappo O, Klopstock N, Olam D, et al. Molecular mechanisms of liver carcinogenesis in the mdr2knockout mice. Mol Cancer Res 2007;5:1159-70.

43. Popov Y, Patsenker E, Fickert P, Trauner M, Schuppan D. Mdr2 (Abcb4)-/- mice spontaneously develop severe biliary fibrosis via massive dysregulation of pro- and antifibrogenic genes. J Hepatol 2005;43:1045-54.

44. Arvola A, Forsander O. Comparison between water and alcohol consumption in six animal species in free choice experiments. Nature 1961;191:819-20.

45. Holmes RS, Dudley JA, Algar EM, Mather PB, Rout UK. Biochemical and genetic studies on enzymes of alcohol metabolism: the mouse as a model organism for human studies. Alcohol Alcohol 1986;21:41-56.

46. Bertola A. Rodent models of fatty liver diseases. Liver Res 2018;2:3-13.

47. Friemel J, Frick L, Unger K, Egger M, Parrotta R, et al. Characterization of HCC mouse models towards an etiology-oriented subtyping approach. Mol Cancer Res 2019;17:1493-502.

48. Ambade A, Satishchandran A, Gyongyosi B, Lowe P, Szabo G. Adult mouse model of early hepatocellular carcinoma promoted by alcoholic liver disease. World J Gastroenterol 2016;22:4091-108.

49. Rafacho BPM, Stice CP, Liu C, Greenberg AS, Ausman LM, et al. Inhibition of diethylnitrosamine-initiated alcohol-promoted hepatic inflammation and precancerous lesions by flavonoid luteolin is associated with increased sirtuin 1 activity in mice. Hepatobiliary Surg Nutr 2015;4:124-34.

50. Brandon-Warner E, Walling TL, Schrum LW, McKillop IH. Chronic ethanol feeding accelerates hepatocellular carcinoma progression in a sex-dependent manner in a mouse model of hepatocarcinogenesis. Alcohol Clin Exp Res 2012;36:641-53.

51. Naugler WE, Sakurai T, Kim S, Maeda S, Kim K, et al. Gender disparity in liver cancer due to sex differences in MyD88-dependent IL-6 production. Science 2007;317:121-4.

52. De Minicis S, Kisseleva T, Francis H, Baroni GS, Benedetti A, et al. Liver carcinogenesis: rodent models of hepatocarcinoma and cholangiocarcinoma. Dig Liver Dis 2013;45:450-9.

53. Xin B, Cui Y, Wang Y, Wang L, Yin J, et al. Combined use of alcohol in conventional chemical-induced mouse liver cancer model improves the simulation of clinical characteristics of human hepatocellular carcinoma. Oncol Lett 2017;14:4722-8.

54. Liu K, McCaughan GW. Epidemiology and etiologic associations of non-alcoholic fatty liver disease and associated HCC. Adv Exp Med Biol 2018;1061:3-18.

55. Wolf MJ, Adili A, Piotrowitz K, Abdullah Z, Boege Y, et al. Metabolic activation of intrahepatic CD8+ T cells and NKT cells causes nonalcoholic steatohepatitis and liver cancer via cross-talk with hepatocytes. Cancer Cell 2014;26:549-64.

56. De Minicis S, Agostinelli L, Rychlicki C, Sorice GP, Saccomanno S, et al. HCC development is associated to peripheral insulin resistance in a mouse model of NASH. PLoS One 2014;9:e97136.

57. Hill-Baskin AE, Markiewski MM, Buchner DA, Shao H, DeSantis D, et al. Diet-induced hepatocellular carcinoma in genetically predisposed mice. Hum Mol Genet 2009;18:2975-88.

58. Kanuri G, Bergheim I. In vitro and in vivo models of non-alcoholic fatty liver disease (NAFLD). Int J Mol Sci 2013;14:11963-80.

59. Asgharpour A, Cazanave SC, Pacana T, Seneshaw M, Vincent R, et al. A diet-induced animal model of non-alcoholic fatty liver disease and hepatocellular cancer. J Hepatol 2016;65:579-88.

60. Tsuchida T, Lee YA, Fujiwara N, Ybanez M, Allen B, et al. A simple diet- and chemical-induced murine NASH model with rapid progression of steatohepatitis, fibrosis and liver cancer. J Hepatol 2018;69:385-95.

61. Kishida N, Matsuda S, Itano O, Shinoda M, Kitago M, et al. Development of a novel mouse model of hepatocellular carcinoma with nonalcoholic steatohepatitis using a high-fat, choline-deficient diet and intraperitoneal injection of diethylnitrosamine. BMC Gastroenterol 2016;16:61.

62. Park EJ, Lee JH, Yu GY, He G, Ali SR, et al. Dietary and genetic obesity promote liver inflammation and tumorigenesis by enhancing IL-6 and TNF expression. Cell 2010;140:197-208.

63. Liang JQ, Teoh N, Xu L, Pok S, Li X, et al. Dietary cholesterol promotes steatohepatitis related hepatocellular carcinoma through dysregulated metabolism and calcium signaling. Nat Commun 2018;9:4490.

64. Henderson JM, Polak N, Chen J, Roediger B, Weninger W, et al. Multiple liver insults synergize to accelerate experimental hepatocellular carcinoma. Sci Rep 2018;8:10283.

65. Castro RE, Diehl AM. Towards a definite mouse model of NAFLD. J Hepatol 2018;69:272-4.

66. Fujii M, Shibazaki Y, Wakamatsu K, Honda Y, Kawauchi Y, et al. A murine model for non-alcoholic steatohepatitis showing evidence of association between diabetes and hepatocellular carcinoma. Med Mol Morphol 2013;46:141-52.

67. Febbraio MA, Reibe S, Shalapour S, Ooi GJ, Watt MJ, et al. Preclinical models for studying NASH-driven HCC: how useful are they? Cell Metab 2019;29:18-26.

68. Takakura K, Koido S, Fujii M, Hashiguchi T, Shibazaki Y, et al. Characterization of non-alcoholic steatohepatitis-derived hepatocellular carcinoma as a human stratification model in mice. Anticancer Res 2014;34:4849-55.

69. Arfianti E, Larter CZ, Lee S, Barn V, Haigh G, et al. Obesity and diabetes accelerate hepatocarcinogenesis via hepatocyte proliferation independent of NF-kB or Akt/mTORC1. J Clin Transl Res 2016;2:26-37.

70. Teufel A, Itzel T, Erhart W, Brosch M, Wang XY, et al. Comparison of gene expression patterns between mouse models of nonalcoholic fatty liver disease and liver tissues from patients. Gastroenterology 2016;151:513-25. 
71. Nakagawa H, Umemura A, Taniguchi K, Font-Burgada J, Dhar D, et al. ER stress cooperates with hypernutrition to trigger TNFdependent spontaneous HCC development. Cancer Cell 2014;26:331-43.

72. Shalapour S, Lin XJ, Bastian IN, Brain J, Burt AD, et al. Inflammation-induced IgA+ cells dismantle anti-liver cancer immunity. Nature 2017;551:340-5.

73. Molina-Sánchez P, Lujambio A. Iron overload and liver cancer. J Exp Med 2019;216:723.

74. Zhou XY, Tomatsu S, Fleming RE, Parkkila S, Waheed A, et al. HFE gene knockout produces mouse model of hereditary hemochromatosis. Proc Natl Acad Sci U S A 1998;95:2492-7.

75. Pigeon C, Turlin B, Iancu TC, Leroyer P, Lan JL, et al. Carbonyl-ironsupplementationinduces hepatocyte nuclear changes in BALB/CJ male mice. J Hepatol 1999;30:926-34.

76. Rothenberg BE, Voland JR. $\beta 2$ knockout mice develop parenchymal iron overload: a putative role for class I genes of the major histocompatibility complex in ironmetabolism. Proc Natl Acad Sci U S A 1996;93:1529-34.

77. Muto Y, Moroishi T, Ichihara K, Nishiyama M, Shimizu H, et al. Disruption of FBXL5-mediated cellular iron homeostasis promotes liver carcinogenesis. J Exp Med 2019;216:950-65.

78. Tripathi A, Debelius J, Brenner DA, Karin M, Loomba R, et al. The gut-liver axis and the intersection with the microbiome. Nat Rev Gastroenterol Hepatol 2018;15:397-411.

79. Yu LX, Schwabe RF. The gut microbiome and liver cancer: mechanisms and clinical translation. Nat Rev Gastroenterol Hepatol 2017; 14:527-39.

80. Yoshimoto S, Loo TM, Atarashi K, Kanda H, Sato S, et al. Obesity-induced gut microbial metabolite promotes liver cancer through senescence secretome. Nature 2013;499:97-101.

81. Achiwa K, Ishigami M, Ishizu Y, Kuzuya T, Honda T, et al. DSS colitis promotes tumorigenesis and fibrogenesis in a choline-deficient high-fat diet-induced NASH mouse model. Biochem Biophys Res Commun 2016;470:15-21.

82. Dapito DH, Mencin A, Gwak GY, Pradere JP, Jang MK, et al. Promotion of hepatocellular carcinoma by the intestinal microbiota and TLR4. Cancer Cell 2012;21:504-16.

83. Poutahidis T, Varian BJ, Levkovich T, Lakritz JR, Mirabal S, et al. Dietary microbes modulate transgenerational cancer risk. Cancer Res 2015;75:1197-204.

84. Llopis M, Cassard AM, Wrzosek L, Boschat L, Bruneau A, et al. Intestinal microbiota contributes to individual susceptibility to alcoholic liver disease. Gut 2016;65:830-9.

85. Xu W, Liu K, Chen M, Sun JY, McCaughan GW, et al. Immunotherapy for hepatocellular carcinoma: recent advances and future perspectives. Ther Adv Med Oncol 2019;11:1758835919862692. 